\title{
Numerical and experimental estimation of thermomechanical fields heterogeneity at the grain scale of 316L stainless steel
}

\author{
Rian Seghir ${ }^{\text {a,b,d }}$, Laurence Bodelot ${ }^{\mathrm{e}}$, Eric Charkaluk ${ }^{\mathrm{a}, \mathrm{b}, \mathrm{d}, *}$, Philippe Dufrénoy ${ }^{\mathrm{a}, \mathrm{c}, \mathrm{d}}$ \\ a Univ Lille Nord de France, F-59000 Lille, France \\ ${ }^{\mathrm{b}}$ ECLille, LML, F-59650 Villeneuve d'Ascq, France \\ ${ }^{\mathrm{c}}$ USTL, LML, F-59650 Villeneuve d'Ascq, France \\ d CNRS, UMR 8107, F-59650 Villeneuve d'Ascq, France \\ e California Institute of Technology, Pasadena, CA, USA
}

\begin{abstract}
Polycrystalline metallic materials are made of an aggregate of grains more or less well oriented with respect to the loading axis. During mechanical loading, the diversity of grain orientations leads to heterogeneous deformation and it is well known that most of the plastic work generated during the deformation process reappears in the form of heat whereas a certain proportion remains latent in the material and is associated with microstructure changes. To access the local stored energy, experimental and numerical energy balances are needed at a suitable scale. In this way experiments have already been done in-house on 316L stainless steel to monitor the evolution of temperature and deformation fields at the microstructural scale. The aim of the present study is now to develop a simplified numerical model, based on experimental observations and able to simulate the thermomechanical behavior, in order to provide a first assessment of the stored energy level and heterogeneity. Our model renders well the average thermomechanical behavior of the specimen and reveals a high heterogeneity of the stored energy at the microstructure scale. Better knowledge of the stored energy at this scale should improve our understanding of strain localization mechanisms.
\end{abstract}

\section{Introduction}

Polycrystalline metallic materials are made of an aggregate of grains more or less well oriented for plastic slip with respect to the loading axis. During mechanical loadings, this diversity of grain orientation leads to inhomogeneous deformation and results in local plasticity. These general aspects are, for example, extensively described in the well-known book of [13]. Additionally, the fact that deformation in polycrystals is inhomogeneous has been first emphasized by [2,7]. During the deformation, this local and then global plasticity triggers a dissipation and a temperature increase of the specimen. The phenomenon has been studied very early by Taylor and co-workers $[17,42,36]$. It is thereby well-known that during cold-working, most of the plastic work reappears in the form of heat whereas a certain proportion remains latent in the material and is associated with microstructure changes, including mainly dislocations elastic energy. An extensive review of the early work within this field was published by [6]. The first estimation of latent energy or stored energy of cold-working was performed by

\footnotetext{
* Corresponding author at: Univ Lille Nord de France, F-59000 Lille, France. Tel.: +33 3206760 86; fax: +33 320335393 .

E-mail address: eric.charkaluk@ec-lille.fr (E. Charkaluk).
}

subtracting the dissipation to the macroscopic work given to the sample. At this time, dissipation during plastic work was approximated thanks to the mean temperature evolution of the whole sample measured by a calorimeter. They concluded that the fraction of plastic work converted into heat could be considered as a constant between 0.8 and 0.95 . Henceforth, most thermomechanical models implemented in finite element codes assume this ratio to be constant. Since then, many attempts have been made to access energy sources using infrared radiometers or cameras $[12,20,25,34]$. Their results, obtained at a macroscopic scale, have shown that the fraction of plastic work stored in the material is not a constant parameter. It actually depends on the strain, the strain rate [26], the class of material and microstructure [35] and the type of loading [33]. In addition, some authors have shown that the stored energy could be a better indicator to predict shear failure than a traditional strain criterion [37] and some works have proven that thermal and heat source measurements could provide crucial information about many macroscopic localization phenomena such as: (1) Lüders bands [22], (2) plastic dissipation in Al aggregates [23], (3) Portevin-Le Chatelier localization [24], and (4) localized phase transformation in Nitinol shape memory alloys [40]. Therefore, it seems possible to track the potential zones where plasticity and damage preferentially develop thanks to the 
evaluation of the kinetics of the dissipation or of the stored energy, as well as the detection of a threshold in those values.

In this continuity, it seems relevant to extend heat sources measurements in order to understand microscale plastic localization phenomena occuring in metals (microplasticity, fatigue, damage) $[29,10,15,38]$ and in order to build fully physically-based constitutive laws and strain localization criteria. However these localization processes lead to very low levels of temperature and occur at a very local scale, thus making any experimental measurement of stored energy laborious and complicated. As a result, to this day, no author has performed an energy balance at the microstructure scale of a polycrystal using full-field measurements and the heat equation, even if some first steps were done on oligocrystals ( $<10$ grains) $[1,38]$. In this work, no experimental energy balance on polycrystals is presented but rather, a first numerical estimation of the local stored energy level and heterogeneity is made, based on experimental data $[8,9]$. The main objective of the work is to study microplastic phenomena, occurring at the grain scale in a polycrystal at low strain level (i.e. $<10 \%$ ) in an overquenched A316L austenitic stainless steel.

This paper conducts, on the one hand, (i) a first comparison between experimental thermal and strain distributions obtained under monotonic tensile test and numerical results based on a simplified description of the sample's texture and constitutive law. On the other hand, it provides (ii) a first estimation of the local stored energy, still hardly experimentally accessible without resorting to strong assumptions [5]. In a first part, the experimental setup capable of measuring kinematic and thermal field at the grain scale is introduced, along with some results it provided. Secondly, the methodology and the assumptions made to build our simplified numerical model are formulated. Finally, comparisons between experimental and numerical results in terms of mean values and distributions are discussed, and the stored energy obtained through our simplified model is presented.

\section{Experimental part}

The results presented in this section are taken from Bodelot's work [8]. It proposed an original experimental setup able to measure, in the same location and at the same time, kinematic and thermal fields at the microstructural scale. The main features are described in [9].

\subsection{Fully-coupled measurements of kinematic and thermal fields}

Strain fields are obtained thanks to DIC (Digital Image Correlation) performed with Correli ${ }^{L M T}$ (developed at the "Laboratoire de Mécanique et Technologie" of Cachan in France) on images of the deforming sample covered with a speckle pattern. Details about the Correli ${ }^{L M T}$ DIC algorithm are exposed in [19]. The spacial resolution of the kinematic fields (displacements, strains) is $104 \mu \mathrm{m} \times 104 \mu \mathrm{m}$ after performing DIC on $16 \times 16$ pixel subsets of the images, the precision on strain values is $0.1 \%$ and the recording frequency is $7 \mathrm{~Hz}$. Temperature fields are obtained by IRT (Infrared Thermography) at a recording frequency of $140 \mathrm{~Hz}$, with a spatial resolution of $90 \mu \mathrm{m} \times 90 \mu \mathrm{m}$ for the temperature fields (after numerical smoothing). In this study, the link between the infrared radiation received by the camera and the temperature of the object is established thanks to a blackbody through a calibration procedure applied on each detector of the camera and leading to a thermal resolution of $0.03^{\circ} \mathrm{C}$.

\subsection{Principle of the coupling}

Since we are interested in the microstructure scale and since the microstructure is different from one face or location to the other on a flat specimen, the same area of the sample has to be observed simultaneously by both cameras. To achieve such a fullycoupled observation of the same zone, a dichroic mirror is placed in front of the sample, making an angle of $45^{\circ}$ with the normal to its surface (cf. Fig. 1). Thanks to its filtering properties, the dichroic mirror transmits the infrared radiation towards the infrared camera, located in front of the sample and reflects the rest of the radiation, including the visible radiation, to the CCD camera which is perpendicular to the normal to the sample. To perform coupled measurements a special coating was designed and applied on the sample, meeting simultaneously requirements of both measurement techniques with the magnifications used. Finally, the DIC
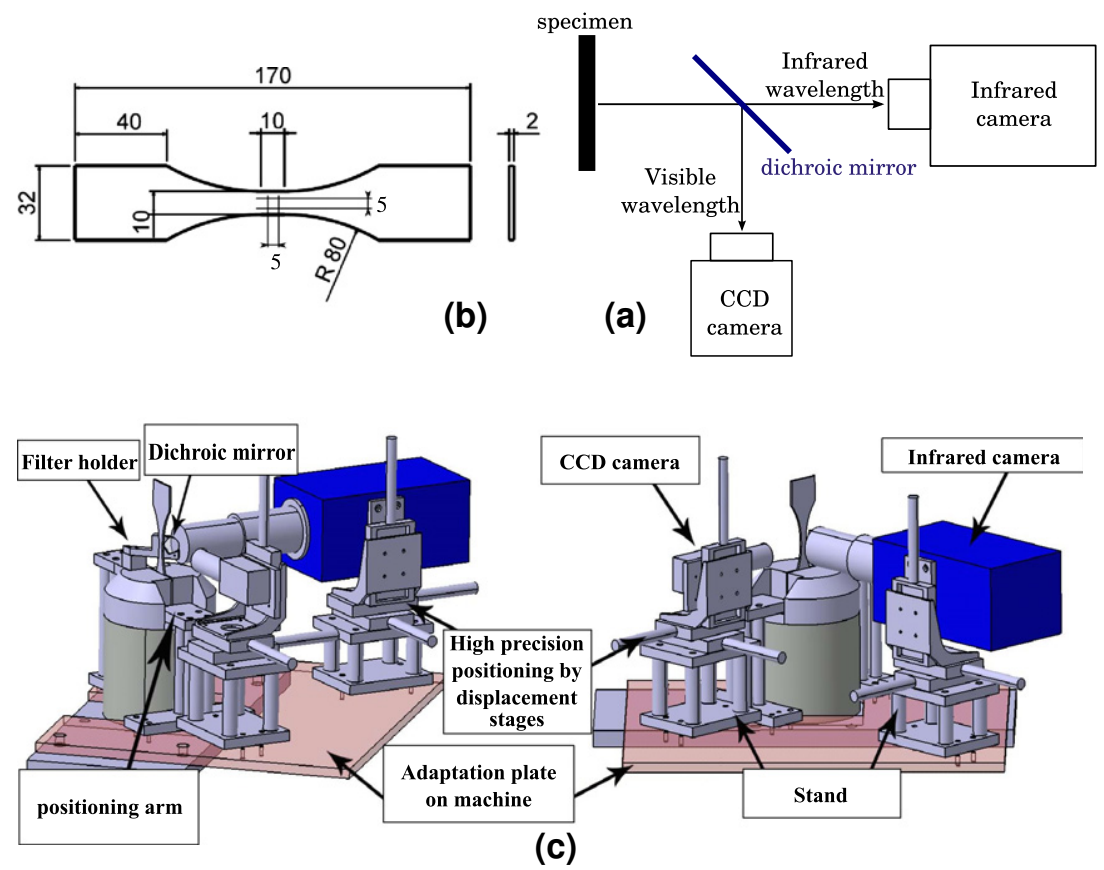

Fig. 1. (a) Flat specimen of stainless steel AISI $316 \mathrm{~L}$ (dimensions in millimeters), (b) schematics of the experimental setup, (c) experimental setup. 


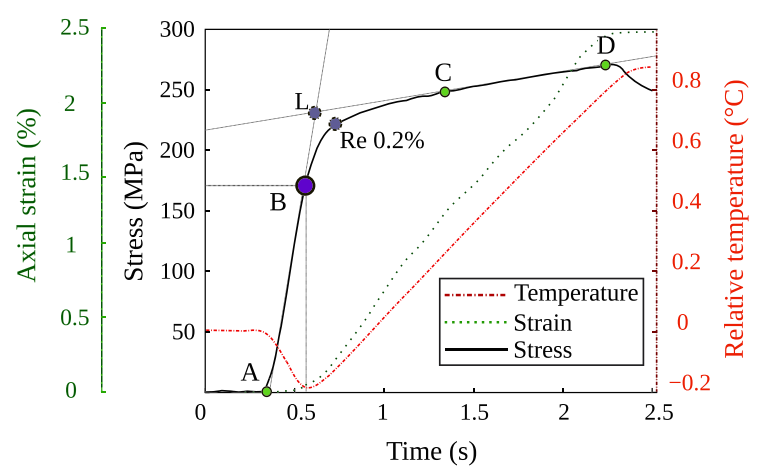

Fig. 2. Mean values (over the studied area) of thermal, strain and stress fields during monotonic tensile test up to $2.5 \%$ mean strain.

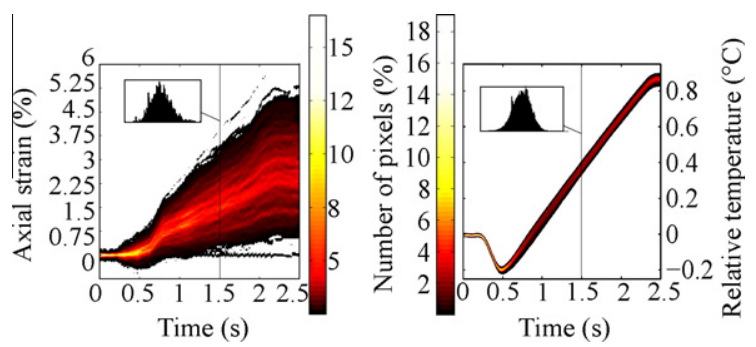

Fig. 3. Distribution of total axial strain and relative temperature during a monotonic tensile test up to $2.5 \%$ mean strain.

displacement data allowed us to track the displacement of material points in front of the infrared array and then express the temperature fields in the undeformed configuration. Therefore the data is fully-coupled insofar as both fields correspond to the same zone, at the same time and are given in the undeformed configuration.

\subsection{Material}

The studied material is an AISI 316L austenitic stainless steel. Dog-bone shaped flat samples $2 \mathrm{~mm}$ thick were heat treated under air for $2 \mathrm{~h}$ at $1200^{\circ} \mathrm{C}$ and immediately water-quenched. The mean grain size of the heat-treated material is $130 \mu \mathrm{m}$, matching the spatial resolution capabilities of both techniques. This means that the spatial resolutions achieved in strain measurements as well as in temperature measurements are suitable to give meaningful data at the scale of the grains of the studied material. It must be underlined that the heat treatment introduced some changes in the behavior compared to a standard 316L stainless steel which generally possesses a grain size close to a few tenths of microns. This point will be discussed later.

\subsection{Results under monotonic tensile test}

A displacement controlled monotonic tensile test was applied to the sample with a constant strain rate of $5 \times 10^{-3} \mathrm{~s}^{-1}$ [8]. As previously described, fully-coupled measurements at the microstructure scale were performed during the monotonic test, providing kinematic and thermal fields. However, as a first step, only average quantities over the complete fields and their associated distributions will be analyzed in this paper. The studied fields correspond to a $5 \mathrm{~mm} \times 5 \mathrm{~mm}$ area in the center of the specimen. Due to the mean size of the grains and this surface area, one can estimate that there is approximately 1500 grains in the studied area. ${ }^{1}$

${ }^{1}$ Some recent EBSD analysis has confirmed that there are exactly 1776 grains.
The evolution of the nominal stress is presented in Fig. 2 and will now be discussed. In a first step, the material deforms elastically up to point $\mathrm{B}$, which corresponds approximately to the beginning of a non-linear relation between stress and strain while the linear relation is represented by line A-L. At this stage, experimental strain fields reveal that some grains of the polycrystal already deform plastically whereas the other grains are still elastic (see the strain distributions of Fig. 3a). Then, between B and the conventional yield stress at $0.2 \%$, which is equal to $223 \mathrm{MPa}$, local plasticity extends and becomes even more generalized until D. This leads to an other linear response of the material from $C$ to $D$ which can be associated to a saturated plastic hardening behavior.

The relative temperature evolution (i.e. the gradient temperature from initial equilibrium) and the strain evolution are also presented in Fig. 2. These values are the spatial averages of the corresponding fields over the studied area at each time increment. One can note a drop in temperature between points A and B both located in the conventional elastic domain, followed by a rise between points B and D. Thermodynamically, this corresponds, in a first step, to a thermoelastic coupling and, in a second step, to the effect of a positive dissipation associated with plasticity. This coupled thermomechanical behavior was underlined many years ago [36]. It is nevertheless interesting to note that the inflexion point in the temperature curve occurs at $\mathrm{B}$, which corresponds to the end of linearity of the macroscopic stress-strain. Therefore, it seems that the thermal inflexion point is linked to a microplastic behavior and that the irreversibilities introduced by the microplasticity become prominent well before the conventional yield stress at $0.2 \%$ strain.

The evolutions of the strain, in axial direction, and temperature distributions are plotted against time in Fig. 3. Transverse strain distributions are not presented here. All distributions can be described by Gaussian functions. However, in the case of strains, the Gaussians have a significant standard deviation which increases with the deformation. It is first interesting to look at the strain distribution on point B (see Fig. 2) which corresponds to the instant when the thermal inflexion point occurs. At this point the mean strain is $0.12 \%$ and the distribution ranges from $-0.63 \%$ to $0.84 \%$. Considering the nominal stress at this point (176 MPa) and the Young's modulus (186 GPa) it is therefore possible to estimate the part of elastic grains within the gage section of the specimen.

$\epsilon^{e} \leqslant \frac{\Sigma}{E}$

It appears that about $43 \%$ of grains are still elastic whereas the rest of the grains are plastic. It is in good agreement with the previous point: microplasticity becomes prominent as temperature begins to increase. One can also observe that some grains are in contraction up to $-0.7 \%$ and, because there are no texture effects (i.e. randomly oriented grains), this contraction is certainly due to the effect of grain size and grain morphology distributions. This has already been observed on cyclically loaded duplex stainless steel [3]. One can also underline that, as the mean deformation evolves from $0 \%$ to $2.5 \%$, the associated local strain distribution ranges from $0.5 \%$ to $5 \%$. At the same time, the mean relative temperature evolves from $0 \mathrm{~K}$ to $0.85 \mathrm{~K}$ when the local distribution varies from $5 \mathrm{mK}$ to $300 \mathrm{mK}$. The fact that thermal fields are more homogeneous is partly due to the thermal diffusivity of material.

It has been shown by observation of the microstructure after the test, that: (i) the zones of highest strains correspond to the more deformed grains (bearing a lot of slip lines), (ii) the zones with the highest temperatures, though more widespread due to diffusion, could be linked to regions where the density of very deformed grains is the most significant at the end of the test [8]. In this study, we will further focus on the mean values of thermal 
and strain fields and on their deviations in order to analyze the relative heterogeneity of the mechanical behavior and in order to give a first numerical estimate of local energy sources during a plastic deformation process.

\section{Numerical estimation of local heterogeneities}

\subsection{Methodology}

Some authors have tried to understand the influence of the constitutive law on the evolution of temperature and on the storage of energy by trying to perform numerical energy balances. One interesting result is that the traditional necessary conditions of mechanical and thermodynamical admissibilities are not sufficient to well predict the thermal evolution and thus, the storage of energy [43]. Therefore, it is fundamental to have a better understanding of thermomechanical couplings if one wants to build a physically based constitutive law. Contrary to experimental investigations, numerical tools always permit to approach unmeasurable quantities. One could distinguish two main approaches: (i) macroscopic approaches, focusing on the description of the mean constitutive law and thus leading to a mean evolution of sources $[43,18]$ and (ii) local approaches, based on crystalline plasticity framework, with different levels of complexity in the description of the constitutive law [38]. The proposed model is midway between both approaches since it tends to render the local heterogeneities without either entering the crystalline plasticity framework or considering phenomenological constitutive laws.

As it was described in the introduction, polycrystalline metallic materials are made of an aggregate of grains. A microstructure possesses many kind of heterogeneities among which only three major ones are underlined here: the local crystallographic orientation of each grain, the grain size and the texture heterogeneity, i.e. specific relations between relative orientations of neighboring grains. This would imply the definition of a polycrystalline aggregate model taking into account these three different aspects [16] and thereby giving stress and strain tensors fields in every grain. However, the definition of such a model is not a simple task and our main goal here is to model thermomechanical couplings at the microscopic scale in order to access a first numerical estimation of the heterogeneities of the stored energy. For this purpose, several assumptions concerning microplastic activity have been made:

- $H_{1}$ : Homogeneous and equiaxed grain shape. A constant mean grain size is assumed and is modeled by a cubic finite element. The chosen size is $150 \mu \mathrm{m} \times 150 \mu \mathrm{m} \times 150 \mu \mathrm{m}$ which is close to the experimental mean size. Thus the grain morphology and size distributions are not taken into account.

- $\mathrm{H}_{2}$ : No crystallographic "texture". No preferential orientation relations between grains are considered. Every grain orientation is independent of its neighbors. Thus, the distribution of crystallographic orientations in space is totally random.

- $H_{3}$ : Plasticity modeling. The macroscopic stress $\Sigma$ necessary for the grain plasticity activation depends, in particular, on the orientation of each grain with respect to the loading axis. At the grain scale, the plastic criterion is generally based on the Schmid theory: $\tau^{i} \leqslant \tau_{c}(i=1, \ldots, 12$ in the case of F.C.C. structure) with the resolved shear stress of the considered slip system $\tau=\Sigma: \boldsymbol{M}, \boldsymbol{M}=\frac{1}{2}(\underline{\boldsymbol{n}} \otimes \underline{v}+\underline{v} \otimes \underline{n}) . \boldsymbol{M}$ is the Schmid matrix, $\underline{n}$ the unit normal vector, $\underline{v}$ the unit slip direction vector of the considered slip system $(i)$ and $\tau_{c}$ a critical shear stress, commonly supposed constant. As $\boldsymbol{M}$ is a function of the orientation of each grain, the distribution of grain orientations in the specimen leads to a $\tau$ distribution for a given $\Sigma$. Assumption $\left(H_{3}\right)$ consists in replacing this $\tau$ distribution by a $\sigma_{y}$ distribution, where $\sigma_{y}$ is a von Mises equivalent yield stress as: $\Sigma_{e q} \leqslant \sigma_{y}$. This distribution relies on experimental distributions of strains. Schmid theory and its construction will be developed in the next paragraph.

\subsection{Local constitutive law}

In the following, the Generalized Standard Material framework [32] is adopted. This framework reduces the construction of the constitutive law to the definition of two potentials: (i) the free energy potential $\Psi$ which describes the local state at every time $t$ and (ii) the dissipation potential $\phi$ which gives the flow rules of the system according to the first and second thermodynamic principles. All the details can be found in Appendix A. Only the main features are described here in order to define the plastic dissipation and the thermoelastic coupling associated with the present model. This model corresponds to an isotropic elasticity, a linear thermoelastic coupling and a linear plastic kinematic hardening. The plastic criterion corresponds to the von Mises yield function which is:

$$
\left\{\begin{array}{l}
f(\boldsymbol{\sigma})=\sqrt{\frac{3}{2} J_{2}(\boldsymbol{s}-\boldsymbol{X})}-\sigma_{y} \\
\boldsymbol{s}=\boldsymbol{\sigma}-\frac{1}{3} \operatorname{tr}(\boldsymbol{\sigma}) \boldsymbol{I} \\
J_{2}(\boldsymbol{s}-\boldsymbol{X})=\frac{1}{2}(\boldsymbol{s}-\boldsymbol{X})(\boldsymbol{s}-\boldsymbol{X}) \\
\boldsymbol{X}=\frac{2}{3} H \boldsymbol{\varepsilon}^{p}
\end{array}\right.
$$

With respect to the first and second thermodynamic principles, the coupled heat equation can be obtained [27] in the present particular modeling framework. Then, by deriving the free energy potential and the plastic dissipation potential [30], the following form is obtained:

$\rho C_{v} \dot{T}-\operatorname{div}(\boldsymbol{K}: \underline{\operatorname{grad}}(T))=r+(\boldsymbol{\sigma}-\boldsymbol{X}): \dot{\boldsymbol{\varepsilon}}^{p}-\alpha T \operatorname{tr}(\dot{\boldsymbol{\sigma}})-9 K \alpha^{2} T \dot{T}$

Three heat sources are present on the left side term:

- $r$ stands for the external sources.

- $(\boldsymbol{\sigma}--\boldsymbol{X}): \boldsymbol{\varepsilon}^{p}$ corresponds to the intrinsic dissipation and is denoted $\dot{d}$.

- $-\alpha \operatorname{Ttr}(\dot{\boldsymbol{\sigma}})-9 K \alpha^{2} T \dot{T}$ corresponds to the thermoelastic coupling.

As external sources are not present in this study, the heat sources are then denoted:

$\dot{\boldsymbol{\Phi}}=(\boldsymbol{\sigma}-\boldsymbol{X}): \dot{\boldsymbol{\varepsilon}}^{p}-\alpha \operatorname{Ttr}(\dot{\boldsymbol{\sigma}})-9 K \alpha^{2} T \dot{T}$

Moreover, as introduced by Taylor [42], the ratio between the stored power and the total plastic power can be defined as:

$\beta=1-\frac{\dot{d}}{\boldsymbol{\sigma}: \dot{\boldsymbol{\varepsilon}}^{p}}$

The numerical implementation of such a thermoplastic framework is also described in Appendix A.

\subsection{Identification of elasto-plastic parameters}

This part presents the different assumptions made in order to identify the mechanical and thermal parameters of the numerical model. As it was previously introduced, the constitutive law is considered as bilinear: linear elasticity followed by linear hardening. The first assumption is to consider the local scale elastoplastic behavior the same as the macroscopic one in terms of moduli. Therefore, the Young's $(E)$ and hardening $(H)$ moduli are identified on an experimental macroscopic tensile strain-stress curve (cf. Fig. 2) and their values are respectively $E=186,000 \mathrm{MPa}$ and $H=1500 \mathrm{MPa}$. 

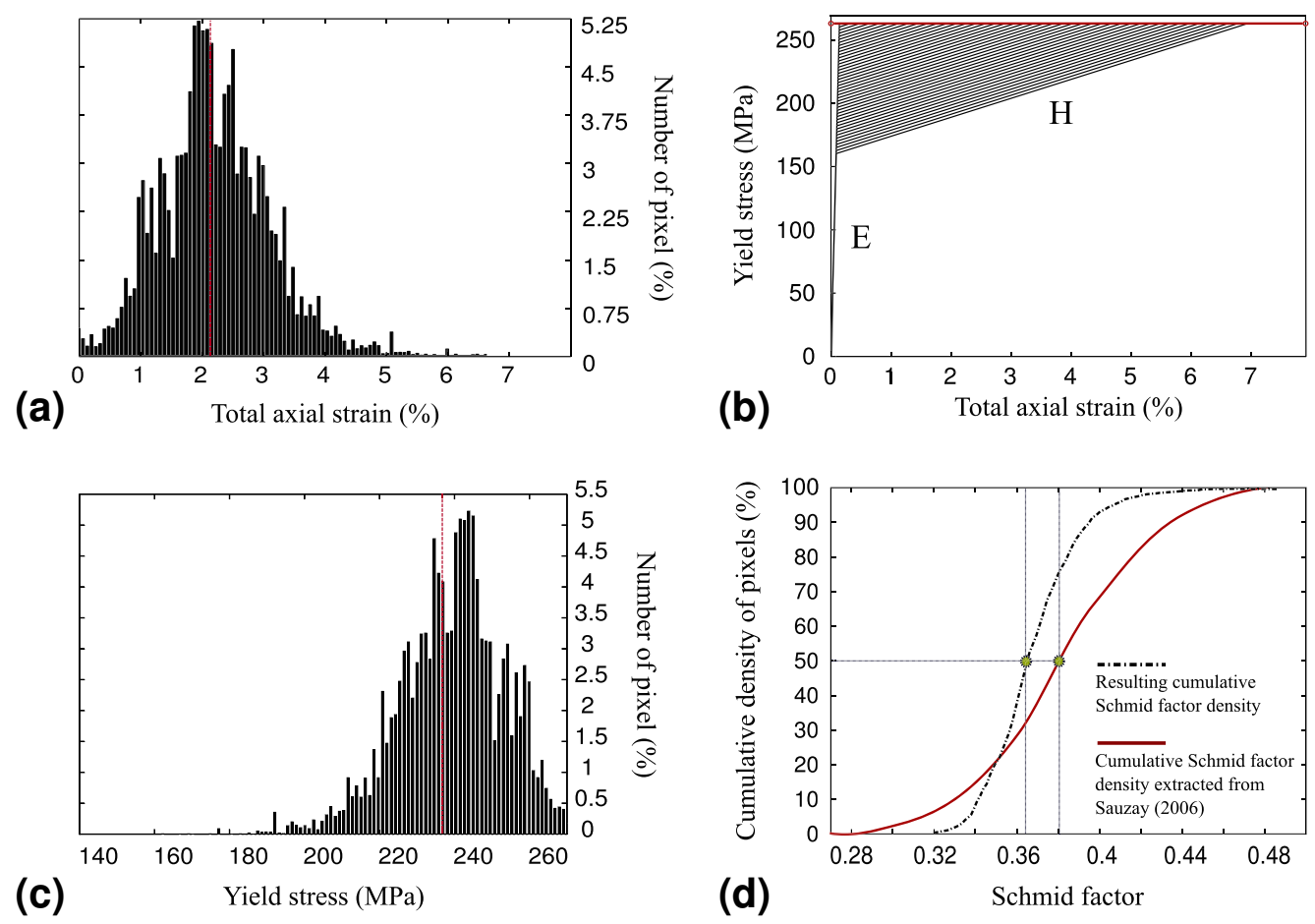

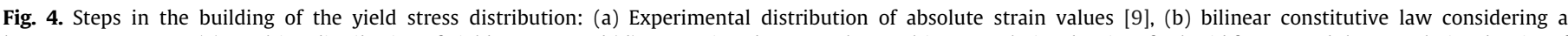

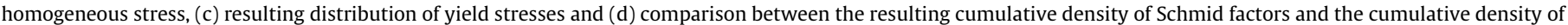
the effective Schmid factors for austenite from Sauzay's works [39].

A relationship between macroscopic and mesoscopic stresses is also necessary. The transition between the macroscopic and mesoscopic stresses can be described in a general case by the following equation:

$\boldsymbol{\sigma}=\boldsymbol{\Sigma}-\mathbb{C}^{*}: \boldsymbol{\varepsilon}^{p}$

It can then be simplified [28] according to some assumptions, in particular:

- Lin-Taylor's model: $\mathbb{C}^{*}=\mathbb{C}$

- Sachs's model: $\mathbb{C}^{*}=0$

where $\mathbb{C}$ is the stiffness tensor of the bulk and $\varepsilon^{p}$ the plastic strain at the local scale.

The first one assumes the homogeneity of the strain $\boldsymbol{\varepsilon}$ in the specimen whereas the second one assumes the homogeneity of the stress $\boldsymbol{\sigma}$. Both these homogenized models are popular because they represent respectively an upper and lower bound to the elastoplastic response of the polycrystal [28]. However, as previously shown in the experimental part of this paper, the total strain is clearly not homogeneous. Therefore, the Lin-Taylor's model could not be considered here and the Sach's one is chosen. As it is a lower bound the elastoplastic response of the polycrystal, it will give a first estimate of the local stresses. Now, as: (i) the experimental distribution of strains at a fixed macroscopic stress is known, (ii) both elastic and hardening moduli are fixed, (iii) the local stresses are assumed homogeneous and equal to the macroscopic one (Sachs's model), a distribution of equivalent von Mises yield stresses can be determined (cf. Fig. 4). The corresponding equations are the following:

$$
\left\{\begin{array}{l}
\sigma_{y}^{g}=\frac{E}{E-H}\left[\Sigma-H \varepsilon_{\Sigma}^{g}\right] \\
\tau_{c}=f^{g} \sigma_{y}^{g}
\end{array}\right.
$$

with $f^{g}$ the Schmid factor of the considered grain $g, \Sigma$ the nominal macroscopic axial stress, $\varepsilon_{\Sigma}^{g}$ the distribution of strains in the central studied area at fixed macroscopic stress $\Sigma, \tau_{c}$ the critical shear stress, which is an intrinsic property of the crystal, and $\sigma_{y}{ }^{g}$ the distribution of yield stresses. The determination of this yield stress distribution follows four successive steps described in Fig. 4:

- in order to take into account the generalized plastic state in the studied zone, the macroscopic stress level is taken at $265 \mathrm{MPa}$, which corresponds to point D in Fig. 2. Then, the corresponding distribution of total strains is plotted in Fig. 4a

- the strain value at each pixel of the observed area is linked to a specific $\sigma_{y}{ }^{g}$ through the bilinear constitutive law deduced from Eq. (7) and from the values of $E, H$ and $\Sigma$ (see Fig. 4b),

- the resulting distribution of yield stresses is plotted in Fig. 4c. This yield stress distribution is included between 170 and $265 \mathrm{MPa}$. This result is qualitatively consistent with the positions of points $B$ and $C$ in Fig. 2, which respectively correspond to the end of the elastic domain and the beginning of perfectly linear plastic domain.

- finally, by taking into account Eq. (7) and a particular value of $\tau_{c}$, the cumulative density of effective Schmid factor is computed and compared with results coming from [39] (cf. Fig. 4d).

The cumulative Schmid factor distributions as defined by [39] could be directly compared with our numerical cumulative density of Schmid factors. As a matter of fact, according to [39], the effective Schmid factor is defined as $f=\max \left(\left|\tau_{i}\right|\right) / \Sigma_{x x}$ where $\Sigma_{x x}$ denotes the macroscopic axial tensile stress and $\max \left(\left|\tau_{i}\right|\right)$ the effective maximum resolved shear stress. Here, the main difference with the classical Schmid theory is that the resolved shear stress is computed considering elastic and plastic crystalline anisotropies. Indeed, in classical Schmid theory, the link between macroscopic stress and resolved shear stress is based on the uniaxiality assumption and, therefore, is directly based on grain orientations. This is true in the case of a single crystal but, in the case of a polycrystal, a structure effect should be considered. In fact, since each grain undergoes multiaxial loading, the resolved shear stress does not 


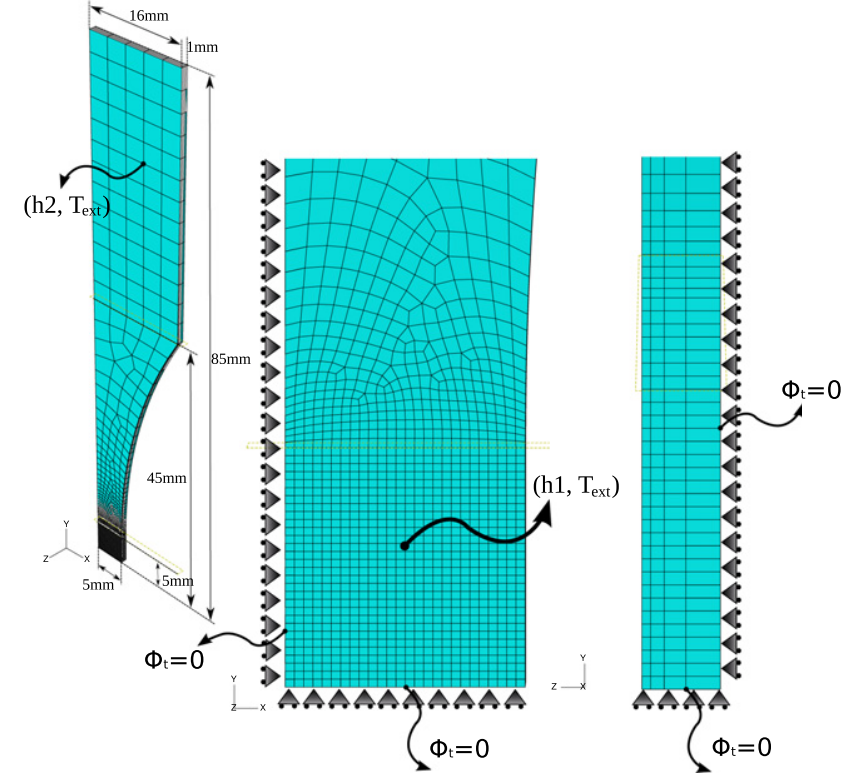

Fig. 5. F.E. model and its boundary conditions.

only depend on the orientation of the grain with respect to the macroscopic tensile axis, but also on crystallographic texture (neighboring grains) and on material anisotropy. Obviously, the experimental strain field intrinsically takes into account crystallographic texture and material anisotropy. Therefore, a cumulative Schmid factor distribution based on experimental data can be directly compared to "the effective" Schmid factor distribution given by [39].

The critical shear stress value chosen is $\tau_{c}=85 \mathrm{MPa}$ and it conducts to resulting Schmid factors ranging between 0.3 and 0.5 , with a mean value of 0.365 . Besides, one can observe that the obtained Schmid factor distribution (cf. Fig. 4d) exhibits a plateau for values lower than 0.32 and greater than 0.45 . The main consequence is that only a few grains possess a very low or a very high Schmid factor. Fig. 4d shows that both effective Schmid factor distributions determined in this work and computed in [39] are qualitatively in good agreement, even if the latter predicts more grains with extreme values of Schmid factor.

\subsection{F.E. model}

Fig. 5 shows the geometrical model of the specimen with its associated mesh and boundary conditions. Due to geometrical symmetry, as well as thermal and mechanical symmetries (i.e., respectively the convective exchanges $\phi_{t}=0$ and displacement $U=0$ with $U$ normal to the symmetry plane), only one quarter of the specimen is modeled. Following [14] where the specimen gage section is modeled by a parallelepiped, the exchanges are represented through two coefficients: $h_{1}$ for the specimen part in contact with air (external temperature $T_{\text {ext }}$ ), and, $h_{2}$, for the specimen ends. The values of $h_{1}$ and $h_{2}$ are determined from the geometry of the specimen gage section (parallelepiped) thermomechanical properties and some numerical 3D thermal computations of relaxation curves. As the effective convective term $h_{2}$ includes the contribution of the convection and the conduction etween specimen ends and machine grips, this leads to a very high value with no physical meaning. However, the obtained value is close to the one of [14] and the one used by [4]. This value allows to obtain the thermal evolution observed during the equilibrium recovery. The different thermophysical and material parameters are summarized in Table 1.
Table 1

Mechanical and thermal properties of the $316 \mathrm{~L}$ stainless steel used in the simulation.

\begin{tabular}{lllll}
\hline$E(\mathrm{MPa})$ & $H(\mathrm{MPa})$ & $\sigma_{Y}(\mathrm{MPa})$ & $v$ & $h_{2}\left(\mathrm{~W} \mathrm{~m}^{-2} \mathrm{~K}^{-1}\right)$ \\
\hline 186,000 & 1500 & {$[170 ; 265]$} & 0.3 & 2200 \\
$\rho\left(\mathrm{kg} \mathrm{m}^{-3}\right)$ & $C_{v}\left(\mathrm{~J} \mathrm{~kg}^{-1} \mathrm{~K}^{-1}\right)$ & $K\left(\mathrm{~W} \mathrm{~m}^{-1} \mathrm{~K}^{-1}\right)$ & $\alpha\left(\mathrm{K}^{-1}\right)$ & $h_{1}\left(\mathrm{~W} \mathrm{~m}^{-2} \mathrm{~K}^{-1}\right)$ \\
8000 & 500 & 15 & $1 \times 10^{-5}$ & 14.6
\end{tabular}

In Table $1, \rho$ denotes the mass density, $C_{v}$ the specific heat capacity, $K$ the conductivity, $\alpha$ the thermal expansion coefficient, $v$ Poisson's ratio, $h_{2}$ and $h_{1}$ the convection coefficients between the specimen ends and the machine's grips, and between the specimen and air, respectively.

The finite element model is based on solid linear tetrahedral elements called C3D8T in Abaqus. The C3D8T is a coupled temperature displacement element. It consists of a brick with eight nodes, and four degrees of freedom $(x, y$ and $z$ displacements $u x, u y, u z$ and temperature). The numerical model contains 6600 of these elements with a regular mesh $(150 \mu \mathrm{m} \times 150 \mu \mathrm{m} \times 150 \mu \mathrm{m})$ in the central area corresponding to the zone studied experimentally. As previously specified, this element size correspond to the estimated experimental mean grain size.

Two different simulations are performed: homogeneous and heterogeneous. In the homogeneous one, the mechanical properties are the same in the whole specimen and are directly identified on the macroscopic tensile curve: $E$ and $H$ are obtained as previously described while the macroscopic yield stress corresponds to point $L\left(\sigma_{y}=233 \mathrm{MPa}\right)$ in Fig. 2 and will correspond to point $E_{2}$ in Fig. 6. In the heterogeneous case, the yield stress distribution is included in the central area.

The loading consists in the application of a displacement ramp, from 0 to $400 \mu \mathrm{m}$, on every surface node of the specimen end. This loading models a perfect contact between the machine's grips and the specimen end (no relative displacement). Both simulations are performed with the same loading, and the results are compared to a monotonic tension test carried at constant strain rate until 2.5\% strain and extracted from [9].

\section{Results and discussion}

\subsection{Beginning of plasticity}

As already described in the experimental part, only spatial mean results in the central region are plotted in Fig. 6 in order to compare macroscopic experimental and numerical curves. One can first note that the evolution of the nominal stress is qualitatively the same in all cases and only the transitions between the elastic and plastic domains seem to be different. In the homogeneous case, one can

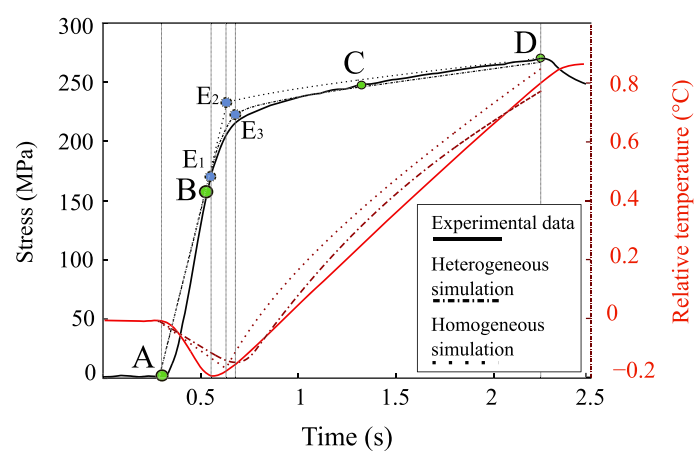

Fig. 6. Mean stress and mean temperature evolutions during monotonic tensile test: comparison with simulation results. 
observe the typical sharp transition of a bilinear elastoplastic constitutive law. In the heterogeneous case, this transition is more progressive even if every local mechanical behavior is also bilinear. Here, plasticity is gradually activated because of the spacial distribution of yield stresses. This heterogeneous simulation results are therefore relatively close to the experimental curve.

The simulated evolution of the spatial mean temperature first exhibits a drop induced by thermoelastic coupling, before a temperature increase due to plastic dissipation. In both homogeneous and heterogeneous cases, the calculated variation of temperature is close to the experimental one but one can note a delay in the calculated elastic-plastic thermal transition. This is due to the existence of a delay in the appearance of plastic strains in each distribution, as will be explained in the next paragraph.

The experimental thermal curve exhibits an inflexion point at $\approx 170 \mathrm{MPa}\left(E_{1}\right)$ whereas the numerical heterogeneous computation gives an inflexion point at $\approx 220 \mathrm{MPa}\left(E_{3}\right)$ and the homogeneous one at $\approx 240 \mathrm{MPa}\left(E_{2}\right)$. This inflexion point in the thermal evolution characterizes the instant when the plastic dissipation resulting of the whole microplasticity becomes prominent. Therefore, this could be considered as a precise measurement of the plastic macroscopic yield stress (see positions of $E_{1}$ and $E_{2}$ on their associated stress curve). Numerical results show that the considered models are not sufficiently refined (grain and texture descriptions, elastoplastic constitutive law, ...) to describe this transition precisely. Fig. 7 confirms this aspect: both numerical simulations conduct to a very homogeneous strain at the beginning of plasticity $\left(E_{1}\right)$ whereas the experimental strain distribution is quite large very early. This lack of local deformation leads to an underestimation of local plasticity and, consequently, to an underestimation of dissipation and temperature. Note that the stress shape at the beginning of the test exhibits a deviation from linearity which is due to the test monitoring. This is not taken into account in the simulation. It could explain the difference of slope of each temperature curve during the drop and probably part of the delay between them.

\subsection{Temperature and strain distributions}

Figs. 7 and 8 present respectively the comparison between experimental and numerical distributions of axial strains and temperatures. Even if the difference between the homogeneous and heterogeneous numerical thermomechanical responses is not so significant in terms of mean values, the distributions are, however, totally different. In the homogeneous case, the strain distribution is almost null and only lead by the specimen geometry and boundary conditions. In comparison, for the heterogeneous simulation, the range of strain is [0.9\%; 3.9\%] at point $\mathrm{D}$ (see Figs. 6 and 7 ) and is obviously due to 'plastic inclusions'. Nevertheless, because of the simplicity of the model, which does not take into account either the real morphology of grains or the local texture, it is not possible to render the experimentally observed contraction in some grains.

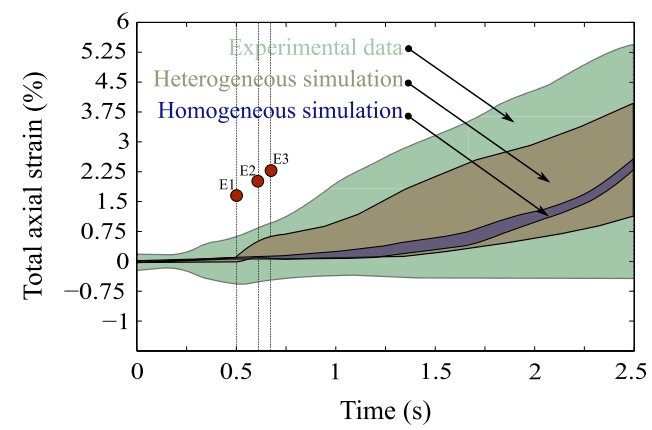

Fig. 7. Strain distribution evolution during monotonic tensile test.

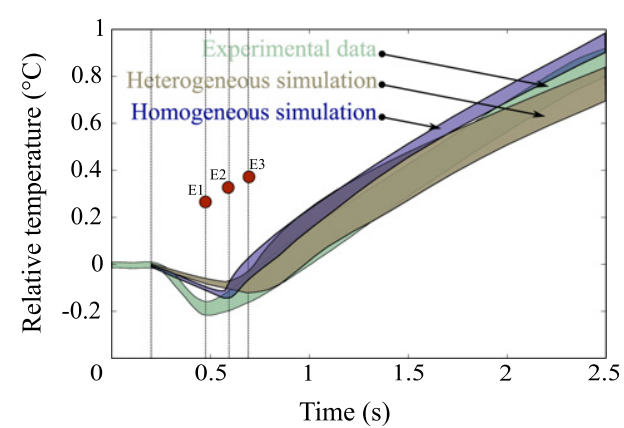

Fig. 8. Thermal distribution evolution during monotonic tensile test.

Moreover, in addition to the same reasons, the differences in effective Schmid factor distributions make the strain distributions extracted from simulations narrower than the experimental ones. Consequently, an underestimation of the local plastic energy levels is expected.

Regarding the thermal distributions, the heterogeneous simulation seems to give a good estimate of the distribution range compared with the experimental one. Nevertheless, this model overestimates the temperature at the beginning of the plastic behavior and finally underestimates it. Such a behavior can also be seen in Fig. 6 through differences in the stress-time curves, where the heterogeneous model also overestimates the stress at the very beginning of the plastic domain and finally underestimates it. The present results put in light the panel of thermal responses which could be provided by different models whereas they all are mechanically admissible as it was already shown in similar studies [43].

\subsection{Dissipated and stored energies}

Since no energy balance has ever been performed on the previous experimental data and since there are only a few published results on this subject, this section presents the comparison between an experimental energy balance performed by [12] and the numerical results provided by the model. The experimental energy balance coming from [12] corresponds to a tensile test from $0 \%$ to $3 \%$ of plastic strain on a standard $316 \mathrm{~L}$ stainless steel, for which a grain size of $8-10 \mu \mathrm{m}$ is expected. Each quantity of this energy balance is calculated using a OD approach and is converted in volumic energy thanks to the knowledge of the "equivalent observation volume" dimensions.

Fig. 9 presents the comparison between numerical and experimental values of dissipated $(D)$ and stored energy $\left(E_{S}\right)$, where:

$$
\begin{aligned}
& D(t)=\int_{0}^{T} \dot{d} d \tau \\
& E_{S}(t)=\int_{0}^{T} \boldsymbol{X}: \dot{\boldsymbol{\varepsilon}}^{p} d \tau
\end{aligned}
$$

where, as previously precised: $\dot{d}=(\boldsymbol{\sigma}-\boldsymbol{X}): \dot{\boldsymbol{\varepsilon}}^{p}$.

Numerical distributions of each quantity are represented by shaded areas while mean experimental results are represented by dashed lines. One can clearly observe a good agreement between the numerical results obtained with the present model and the experimental dissipated energy. The mean value of this energy is about $6 \mathrm{~mJ} / \mathrm{mm}^{3}$ at 3\% plastic strain and the local heterogeneity of numerical dissipated energy range from 4 to $8.5 \mathrm{~mJ} / \mathrm{mm}^{3}$. This is consistent with the previous thermal results.

As far as the stored energy is concerned, one can note that the mean value is underestimated from about a factor 5 . The main difficulty comes from the evolution of the energy storage at the very 


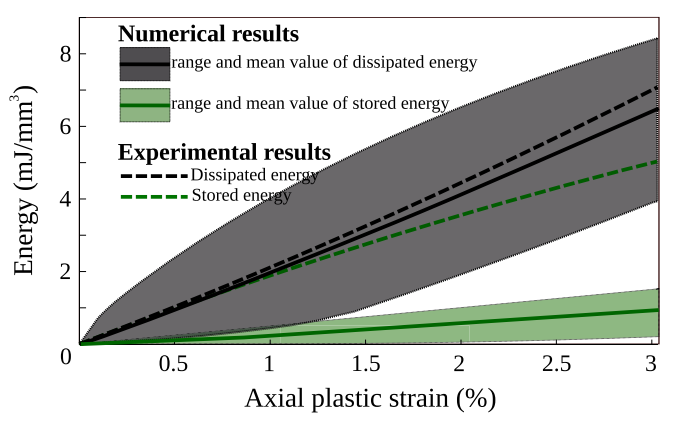

Fig. 9. Comparison between experimentally stored and dissipated energy extracted from [12] (grain size $\approx 10 \mu \mathrm{m}$ ) and their numerical counterpart calculated for a monotonic tensile test (grain size $\approx 130 \mu \mathrm{m}$ ).

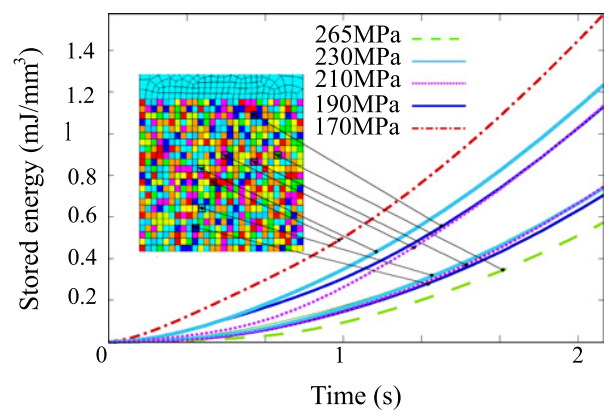

Fig. 10. Distribution of stored energy during monotonic tensile test.

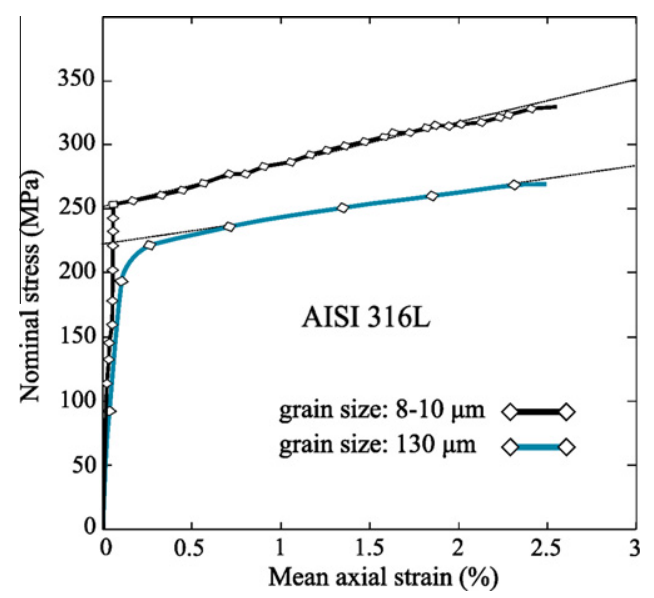

Fig. 11. Stress-strain curve of A316L stainless steel for small grain size [12] and big ones.

beginning of the plastic behavior. Indeed, many works have shown that the ratio between storage and anelastic power $\beta$, is not a constant: it can reach $60 \%$ at the very beginning of plasticity and then tends to 0 when a quasi-perfectly plastic behavior is reached. In the present model, due to simple linearity of the kinematic hardening law, such an evolution cannot be observed. Nevertheless, this comparison of stored energy level must be done while bearing in mind that there are microstructure differences. As already mentioned, results extracted from [12] are based on a conventional AISI316L stainless steel for which surface grain size is about $10 \mu \mathrm{m} \times 10 \mu \mathrm{m}$ whereas grains in the case of the present heat treated $\mathrm{A} 316 \mathrm{~L}$ are about $130 \mu \mathrm{m} \times 130 \mu \mathrm{m}$. It has already been shown in [35] that the grain size is a crucial parameter that influences the storage of energy. Oliferuk's work has been conducted on an austenitic stainless steel and "small" grain $(8 \mu \mathrm{m})$ and "big" grains $(80 \mu \mathrm{m})$ microstructures are compared. It has been shown that for small strains less than $5 \%$, there is a difference close to $30-40 \%$ in the energy storage since small grains store more energy than big grains. This difference decreases as the strain level increases. This is also confirmed by the stress-strain curves plotted in Fig. 11. In the case of "small" grains used in Chrysochoos's study, the yield stress and the hardening are more important than in our case (considered as "big" grains). Consequently, storage of energy must be greater in the Chrysochoos's study. Thus, in addition to a possible numerical underestimation of the stored energy due to the simplicity of the model one can expect-as in the case of Oliferuk-a 30-40\% difference in the storage between Chrysochoos's work and the present work solely because of this grain size effect. This could partly explain the present results. Finally, contrary to existing FE code such as Abaqus, our simplified modeling permits to observe a variation of the stored energy as a function of strain as well as a variation in the rate of storage which could reflect an acceleration of local storage (see Fig. 10).

Further analysis can be done due to the heterogeneous character of the proposed model. Several elements have been selected on the observed face of the specimen and the evolution of their local stored energy is presented in Fig. 10. We show that the existence of local yield stresses is not enough to explain the heterogeneity of energy: neighbors and their behavior do have a great influence, as it was already put in light in similar context [39]. First, one can see that the yield stresses of the elements do not range like their stored energy. As an additional proof, couples of elements with yield stresses of $190 \mathrm{MPa}, 210 \mathrm{MPa}$ and $230 \mathrm{MPa}$ were selected in Fig. 10. Each couple of elements has the same behavior, nevertheless the stored energy for one of them is up to $50 \%$ more than the other one. Yield stresses are totally random in space within the central zone. It is therefore complicated to exhibit macrozone with similar behavior to explain this result but elements which store more energy seem to be mainly surrounded by elements with lower yield stress. Concretely the storage of energy is the result of a competition between the grain own yield stress (grain orientation) and the yield stress of the neighbors (neighboring orientations). We can also observe an heterogeneity in the rate of energy storage. Indeed some grains have a very low rate of energy storage at the beginning of the simulation but thanks to a local increase in the rate of storage, they end up at the same, or at a greater, level of stored energy. This is well-illustrated in Fig. 10 by the 190, 210 and $230 \mathrm{MPa}$ curves: right underneath the $170 \mathrm{MPa}$ curve, the $190 \mathrm{MPa}$ curve diverges from the $230 \mathrm{MPa}$ one to converge towards the $210 \mathrm{MPa}$ curve at the end of the simulation.

\section{Conclusion}

An experimental setup for in situ observation of strain and thermal fields on the same area of a sample at the scale of its microstructure has been developed in a previous work. A monotonic tensile test was performed and the temperature and strain fields at the local level were recorded. To estimate the heterogeneity of energy distribution at this scale and to improve the link between models and thermomechanical microscale observations, a thermo-elasto plastic model was developed. It is based on a distribution of random yield stresses and thermo-elasto-plastic coupled local behavior. This work has lead to a first estimation of the average level of energy stored within a material as well as a first estimation of the stored energy local heterogeneities. 
- The analysis of results, in terms of temperature and strain, shows that a good representation of the local crystallographic texture is a key parameter to render local experimental observations correctly. This is the role of the yield stress distribution included in the model.

- The relatively narrow distribution of temperatures, compared to the broader distribution of stored energy values, implies that temperature fields are more uniform than the stored energy fields. It thus shows the limit of the thermal information. The thermal response is instantaneous and strongly dependent on conduction phenomena and boundary conditions. On the other hand, energy fields contain information about the history of loading, the localization phenomenon, and their kinetics.

- The already strong heterogeneity of the energy field is probably underestimated because of the underestimation of strain heterogeneity.

As different constitutive laws can provide very similar mechanical responses but a variety of thermal responses, analyzing the thermal and energy response could be, in our opinion, a way to discriminate between models. A model closer to reality, based on an experimental measurement of local crystal orientations by EBSD and on a crystalline thermoplasticity model will be developed soon to clarify these initial results and to propose a model of energybased local behavior.

\section{Acknowledgments}

This study has been funded by the CNRS, the Nord-Pas-de-Calais area (CISIT) and the European Community (FEDER).

\section{Appendix A. Thermoelastoplasticity: constitutive law and numerical implementation}

\section{A.1. Thermoplastic framework}

In this appendix, the Generalized Standard Material framework [32] is adopted. This framework reduces the construction of the constitutive law to the definition of two potentials: (i) the free energy potential $\Psi$ which describes the local state at every time $t$ and (ii) the dissipation potential $\phi$ which gives the flow rules of the system according to the first and second thermodynamic principles. $\Psi$ is chosen convex quadratic to ensure that the dissipation remains positive for any system variation. In addition, a partition of the total deformation $\boldsymbol{\varepsilon}$ into an elastic part $\boldsymbol{\varepsilon}^{e}$ and a plastic part $\boldsymbol{\varepsilon}^{p}$ is assumed. Thus, $\Psi$ is written as a function of three variables: the absolute temperature $T, \boldsymbol{\varepsilon}^{e}$ and $\boldsymbol{\varepsilon}^{p}$ :

$\boldsymbol{\varepsilon}=\boldsymbol{\varepsilon}^{e}+\boldsymbol{\varepsilon}^{p}$

$\Psi=\Psi\left(T, \boldsymbol{\varepsilon}^{e}, \boldsymbol{\varepsilon}^{p}\right)$

The dual thermodynamic variables associated to $\boldsymbol{\varepsilon}^{e}$ and $\boldsymbol{\varepsilon}^{p}$ are respectively $\boldsymbol{\sigma}$ and the backstress $\boldsymbol{X}$. Thus:

$\boldsymbol{\sigma}=\rho \frac{\partial \Psi}{\partial \boldsymbol{\varepsilon}^{e}} ; \quad \boldsymbol{X}=\rho \frac{\partial \Psi}{\partial \boldsymbol{\varepsilon}^{p}}$

In the case of an isotropic elasticity, linear thermo-elastic coupling and linear plastic kinematic hardening, the free energy potential $\Psi$ is written [21]:

$\rho \Psi=\left[\frac{1}{2}\left(\lambda \operatorname{tr}\left(\boldsymbol{\varepsilon}^{e}\right)^{2}+2 \mu \operatorname{tr}\left(\boldsymbol{\varepsilon}^{e^{2}}\right)\right)-(3 \lambda+2 \mu) \alpha \theta \operatorname{tr}\left(\boldsymbol{\varepsilon}^{e}\right)+\frac{1}{3} H \operatorname{tr}\left(\boldsymbol{\varepsilon}^{p^{2}}\right)\right]$

with $\lambda$ and $\mu$ the Lamé coefficients, $\alpha$ the thermal expansion coefficient, $\theta=T-T_{0}$ with $T_{0}$ the initial temperature and $H$, the hardening modulus. Note that the thermal expansion of the material is as- sumed to be isotropic. The plastic criterion corresponds to the von Mises yield function which is:

$$
\left\{\begin{array}{l}
f(\boldsymbol{\sigma})=\sqrt{\frac{3}{2} J_{2}(\boldsymbol{s}-\boldsymbol{X})}-\sigma_{y} \\
\boldsymbol{s}=\boldsymbol{\sigma}-\frac{1}{3} \operatorname{tr}(\boldsymbol{\sigma}) \boldsymbol{I} \\
J_{2}(\boldsymbol{s}-\boldsymbol{X})=\frac{1}{2}(\boldsymbol{s}-\boldsymbol{X})(\boldsymbol{s}-\boldsymbol{X}) \\
\boldsymbol{X}=\frac{2}{3} H \boldsymbol{\varepsilon}^{p}
\end{array}\right.
$$

By derivation of Eq. (A.4), one obtains the $\boldsymbol{\sigma}$ tensor:

$\boldsymbol{\sigma}=\rho \frac{\partial \Psi}{\partial \boldsymbol{\varepsilon}^{e}}=\lambda \operatorname{tr}\left(\boldsymbol{\varepsilon}^{e}\right) \boldsymbol{I}+2 \mu \boldsymbol{\varepsilon}^{e}-3 K \alpha \theta \boldsymbol{I}$

with $3 K=3 \lambda+2 \mu$. The total derivative of the stress tensor $\sigma$ could be written:

$\mathrm{d} \boldsymbol{\sigma}=\frac{\partial \boldsymbol{\sigma}}{\partial \boldsymbol{\varepsilon}^{e}}: \mathrm{d} \boldsymbol{\varepsilon}^{e}+\frac{\partial \boldsymbol{\sigma}}{\partial T} \mathrm{~d} T$

Thus, taking into account (A.6):

$\mathrm{d} \boldsymbol{\sigma}=\mathbb{C}: \mathrm{d} \boldsymbol{\varepsilon}^{e}-3 K \alpha \mathbf{I d} T=\mathbb{C}:\left(\mathrm{d} \boldsymbol{\varepsilon}-\mathrm{d} \boldsymbol{\varepsilon}^{p}\right)-3 K \alpha \mathbf{I d} T$

Moreover, with respect to the first and second thermodynamic principles, the coupled heat equation can be obtained [27]:

$$
\begin{aligned}
\rho C_{\nu} \dot{T} & =r+\operatorname{div}(\boldsymbol{K}: \underline{\operatorname{grad}}(T))+\boldsymbol{\sigma}: \dot{\boldsymbol{\varepsilon}}+\rho T \frac{\partial^{2} \Psi}{\partial T \partial \boldsymbol{\varepsilon}^{e}}: \dot{\boldsymbol{\varepsilon}^{e}}-\rho \frac{\partial \Psi}{\partial \boldsymbol{\varepsilon}^{e}} \\
& : \dot{\boldsymbol{\varepsilon}}^{e}+\rho T \frac{\partial^{2} \Psi}{\partial T \partial \boldsymbol{\varepsilon}^{p}}: \dot{\boldsymbol{\varepsilon}^{p}}-\rho \frac{\partial \Psi}{\partial \boldsymbol{\varepsilon}^{p}}: \dot{\boldsymbol{\varepsilon}^{p}}
\end{aligned}
$$

Taking into account the Eq. (A.3) into the heat coupled Eq. (A.9), one obtain:

$$
\begin{aligned}
\rho C_{v} \dot{T} & =r+\operatorname{div}(\boldsymbol{K}: \underline{\operatorname{grad}}(T))+\boldsymbol{\sigma}: \dot{\boldsymbol{\varepsilon}}+\rho T \frac{\partial^{2} \Psi}{\partial T \partial \boldsymbol{\varepsilon}^{e}}: \dot{\boldsymbol{\varepsilon}}^{e}-\boldsymbol{\sigma} \\
& : \dot{\boldsymbol{\varepsilon}^{e}}+\rho T \frac{\partial^{2} \Psi}{\partial T \partial \boldsymbol{\varepsilon}^{p}}: \dot{\boldsymbol{\varepsilon}^{p}}-\boldsymbol{X}: \dot{\boldsymbol{\varepsilon}}{ }^{p}
\end{aligned}
$$

The term $\rho T \frac{\partial^{2} \Psi}{\partial T \partial \varepsilon^{p}}: \dot{\boldsymbol{\varepsilon}}^{p}$ corresponds to the thermoplastic coupling, usually linked to phase changes in the material. In the present case, this term is neglected. Thus, the term $\rho T \frac{\partial^{2} \Psi}{\partial T \partial \varepsilon^{e}}: \dot{\boldsymbol{\varepsilon}}^{e}$ denoted $d_{T e}$, which corresponds to the thermoelastic coupling, is the only coupling term present in the equation. It can be written as follows:

$d_{T e}=T \frac{\partial \boldsymbol{\sigma}}{\partial T}: \dot{\boldsymbol{\varepsilon}}^{e}=-3 K \alpha T \boldsymbol{I}: \dot{\boldsymbol{\varepsilon}}^{e}=-3 K \alpha \operatorname{Ttr}\left(\dot{\boldsymbol{\varepsilon}}^{e}\right)$

As the expression of the elastic strain is the following:

$\boldsymbol{\varepsilon}^{e}=\frac{1+v}{E} \boldsymbol{\sigma}-\frac{v}{E} \operatorname{tr}(\boldsymbol{\sigma}) \boldsymbol{I}+\alpha\left(T-T_{0}\right) \boldsymbol{I}$

The thermoelastic term is:

$d_{T e}=-\alpha \operatorname{Ttr}(\dot{\boldsymbol{\sigma}})-9 K \alpha^{2} T \dot{T}$

By taking into account the heat Eq. (A.10) and by deriving the plastic dissipation potential [30], one obtains:

$\rho C_{v} \dot{T}-\operatorname{div}(\boldsymbol{K}: \underline{\operatorname{grad}}(T))=r+(\boldsymbol{\sigma}-\boldsymbol{X}): \dot{\boldsymbol{\varepsilon}}^{p}-\alpha \operatorname{Ttr}(\dot{\boldsymbol{\sigma}})-9 K \alpha^{2} T \dot{T}$

Three heat sources are present on the left side term:

- $r$ stands for the external sources.

- $(\boldsymbol{\sigma}-\boldsymbol{X}): \dot{\boldsymbol{\varepsilon}}^{p}$ corresponds to the intrinsic dissipation and is denoted $\dot{d}$.

- $-\alpha \operatorname{tr}(\dot{\boldsymbol{\sigma}})-9 K \alpha^{2} T \dot{T}$ corresponds to the thermoelastic coupling.

Then, as external sources are not present in this study, the heat sources are denoted:

$\dot{\boldsymbol{\Phi}}=(\boldsymbol{\sigma}-\boldsymbol{X}): \dot{\boldsymbol{\varepsilon}}^{p}-\alpha \operatorname{Ttr}(\dot{\boldsymbol{\sigma}})-9 K \alpha^{2} T \dot{T}$

Moreover, as introduced by Taylor [42], the ratio between the stored power and the total plastic power can be defined as: 
$\beta=1-\frac{\dot{d}}{\boldsymbol{\sigma}: \dot{\boldsymbol{\varepsilon}^{p}}}$

\section{A.2. Numerical implementation}

In order to perform numerical simulations based on the finite element (FE) method, Abaqus 6.5. commercial package is chosen along with its Abaqus/standard implicit solver. In this code, fully thermomechanical coupled calculations were introduced to solve forming processes problems where the variations of temperature are mainly lead by plastic dissipation. Therefore thermoelastic coupling is not included in the code and the definition of the partition between dissipated and stored energies is relatively poor, basically based on the $\frac{1}{\beta}$ ratio. It is therefore necessary to implement the previous model as a User-defined stress update algorithm, denoted UMat in Abaqus and written in Fortran 77. At each loading increment and for each integration point, the FE code provides the mechanical quantities and the strain increment to the UMat which updates the stresses and all internal variables. Then the UMat computes the mechanical and thermal tangent moduli as well as the coupled moduli, and finally the thermal and plactic strain increments. This scheme introduces a non-symmetric jacobian matrix:

$$
\left(\begin{array}{ll}
\mathbb{K}_{u u} & \boldsymbol{K}_{u \theta} \\
\boldsymbol{K}_{\theta u} & \boldsymbol{K}_{\theta \theta}
\end{array}\right)\left\{\begin{array}{c}
\Delta \underline{u} \\
\Delta T
\end{array}\right\}=\left\{\begin{array}{l}
\underline{R}_{u} \\
R_{\theta}
\end{array}\right\}
$$

where $\mathbb{K}_{u u}, \boldsymbol{K}_{\theta \theta}, \boldsymbol{K}_{\theta u}, \boldsymbol{K}_{u \theta}$ denote the assembly of the local tangent stiffness. ${ }^{2}$ Local tangent stiffness are defined in relations (A.18a-d) respectively. Details about their calculation can be found in [8].

$$
\begin{cases}\frac{\partial \Delta \boldsymbol{\sigma}}{\partial \Delta \varepsilon}=\mathbb{C}-2 \mu \frac{\partial \Delta \varepsilon^{p}}{\partial \Delta \varepsilon} & \text { (a) } \\ \frac{\partial \Delta \boldsymbol{\sigma}}{\partial \Delta T}=-3 K \alpha \boldsymbol{I} & \text { (b) } \\ \frac{\partial \Delta \dot{\Phi}}{\partial \Delta \varepsilon}=(\boldsymbol{\sigma}-\boldsymbol{X}) \frac{\partial \Delta \varepsilon^{p}}{\partial \Delta \varepsilon}-\alpha T \boldsymbol{I}: \frac{\partial \Delta \boldsymbol{\sigma}}{\partial \Delta \varepsilon} & \text { (c) } \\ \frac{\partial \Delta \dot{\Phi}}{\partial \Delta T}=-9 K \alpha^{2} T & \text { (d) }\end{cases}
$$

The incremental form of the Eqs. (A.8) and (A.15) are $^{3}$ :

$$
\begin{aligned}
\Delta \boldsymbol{\sigma} & =\mathbb{C}:\left(\Delta \boldsymbol{\varepsilon}-\Delta \boldsymbol{\varepsilon}^{p}\right)-3 K \alpha \mathbf{I} \Delta T \\
\Delta \dot{\Phi} & =(\boldsymbol{\sigma}-\boldsymbol{X}): \frac{\Delta \boldsymbol{\varepsilon}^{p}}{\Delta t}-\alpha T \operatorname{tr}\left(\frac{\Delta \boldsymbol{\sigma}}{\Delta t}\right)-9 K \alpha^{2} T \frac{\Delta T}{\Delta t}
\end{aligned}
$$

In this incremental problem, the resolution consists in the determination of the plastic strain increment $\Delta \varepsilon^{p}$ eventually induced by a firstly supposed purely elastic stress tensor increment. This plastic strain increment has then to verify the consistency condition (Kuhn-Tucker condition) and the normality rule, which are respectively written:

$$
\left\{\begin{array}{l}
\dot{f}(\boldsymbol{\sigma})=0 \\
\Delta \boldsymbol{\varepsilon}^{p}=\dot{\lambda} \frac{\partial f}{\partial \boldsymbol{\sigma}} \Delta t
\end{array}\right.
$$

The calculation of $\Delta \varepsilon^{p}$ is here based on the radial return mapping method firstly developed by $[31,41]$. More details about this problem can be found in [11]. The proposed implementation is time implicit in displacement and time explicit in temperature and the coupled non linear system is solved by a Newton method.

\section{References}

[1] C. Badulescu, M. Grédiac, H. Haddadi, J.-D. Mathias, X. Balandraud, H.-S. Tran, Mechanics of Materials 43 (1) (2011) 36-53.

[2] C. Barrett, Structure of Metals, McGraw-Hill Book Company, Inc., New York, 1948.

\footnotetext{
${ }^{2}$ Following the Abaqus notations, the different stiffness $\mathbb{K}_{u u}, \boldsymbol{K}_{\theta \theta}, \boldsymbol{K}_{\theta u}, K_{u \theta}$ corresponds to the assembly of the local tangent stiffness (A.18a-d) for each integration point.

${ }^{3}$ Following the Abaqus notations, $\Delta \dot{\Phi}$ correspond to RPL.
}

[3] A. El Bartalli, V. Aubin, S. Degallaix, International Journal of Fatigue 31 (11-12) (2009) 2049-2055.

[4] B. Berthel, Mesures thermographiques de champs de dissipation accompagnant la fatigue à grand nombre de cycles des aciers. Ph.D. thesis, Thèse de doctorat de l'université de Montpellier II, Spécialité Mécanique, 2007. <http://tel.archives-ouvertes.fr/tel-00410074/fr/> (in French).

[5] B. Berthel, B. Wattrisse, A. Chrysochoos, A. Galtier, Strain 43 (3) (2007) 273279.

[6] M.B. Bever, D.L. Holt, A.L. Titchener, Progress in Materials Science 17 (1973) 5177.

[7] W. Boas, M. Hargreaves, Proceedings of the Royal Society A 193 (1032) (1948) 89-97.

[8] L. Bodelot, Etude couplées des champs cinématiques et thermiques à l'échelle de la microstructure des matériaux métalliques, Ph.D. thesis, Thèse de doctorat de l'université de Lille1, Spécialité Mécanique, 2008, <http://tel.archivesouvertes.fr/tel-00347987/fr/> (in French).

[9] L. Bodelot, L. Sabatier, E. Charkaluk, P. Dufrènoy, Materials Science and Engineering: A 501 (1-2) (2009) 52-60.

[10] T. Boulanger, A. Chrysochoos, C. Mabru, A. Galtier, International Journal of Fatigue 26 (3) (2004) 221-229.

[11] E. Charkaluk, A. Constantinescu, Comptes Rendus Mécanique 334 (6) (2006) 373-379.

[12] A. Chrysochoos, J.-C. Chezeaux, H. Caumon, Revue de Physique Appliquée (Paris) 24 (2) (1989) 215-225.

[13] A. Cottrell, Dislocations and Plastic Flow in Crystals, Clarendon Press, Oxford, 1953.

[14] C. Doudard, S. Calloch, P. Cugy, A. Galtier, F. Hild, Fatigue \& Fracture of Engineering Materials \& Structures 28 (2005) 279-288. fFEMS3 Contrat Arcelor.

[15] S. Dumoulin, H. Louche, O. Hopperstad, T. Børvik, European Journal of Mechanics - A/Solids 29 (3) (2010) 461-474.

[16] P. Evrard, A.E. Bartali, V. Aubin, C. Rey, S. Degallaix, D. Kondo, International Journal of Solids and Structures 47 (16) (2010) 1979-1986.

[17] W.S. Farren, G.I. Taylor, Proceedings of the Royal Society of London. Series A, Containing Papers of a Mathematical and Physical Character 107 (743) (1925) 422-451.

[18] P. Håkansson, M. Wallin, M. Ristinmaa, International Journal of Plasticity 21 (7) (2005) 1435-1460.

[19] F. Hild, B. Raka, M. Baudequin, S. Roux, F. Cantelaube, Applied Optics 41 (32) (2002) 6815-6828.

[20] J. Hodowany, G. Ravichandran, A. Rosakis, P. Rosakis, Experimental Mechanics 40 (2000) 113-123.

[21] J. Lemaitre, J.L. Chaboche, Mechanics of Solids Materials, Cambridge University Press, 1994.

[22] H. Louche, A. Chrysochoos, Materials Science and Engineering: A 307 (1-2) (2001) 15-22.

[23] H. Louche, L. Tabourot, Materials Science Forum (2004) 1395-1400.

[24] H. Louche, P. Vacher, R. Arrieux, Materials Science and Engineering: A 404 (12) (2005) 188-196.

[25] D. Macdougall, Experimental Mechanics 40 (2000) 298-306.

[26] M. Maj, W. Oliferuk, Journal of Physics: Conference Series 240 (1) (2010). paper number: 012143.

[27] G. Maugin, The Thermomechanics of Plasticity and Fracture, Cambridge University Press, 1992.

[28] V. Monchiet, E. Charkaluk, D. Kondo, Comptes Rendus Mécanique 334 (2) (2006) 129-136.

[29] A. Morabito, A. Chrysochoos, V. Dattoma, U. Galietti, International Journal of Fatigue 29 (5) (2007) 977-984.

[30] Z. Mróz, W. Oliferuk, International Journal of Plasticity 18 (3) (2002) 379-397.

[31] Q.S. Nguyen, International Journal for Numerical Methods in Engineering 11 (5) (1977) 817-832.

[32] Q.S. Nguyen, B. Halphen, Journal de Mécanique 14 (1975) 39-63.

[33] W. Oliferuk, M. Maj, Materials Science and Engineering A (2004) 218-221.

[34] W. Oliferuk, W.A. Swiatnicki, M.W. Grabski, Materials Science and Engineering: A 161 (1) (1993) 55-63.

[35] W. Oliferuk, W.A. Swiatnicki, M.W. Grabski, Materials Science and Engineering A 197 (1) (1995) 49-58.

[36] H. Quinney, G.I. Taylor, Proceedings of the Royal Society of London. Series A Mathematical and Physical Sciences 163 (913) (1937) 157-181.

[37] D. Rittel, Z.G. Wang, M. Merzer, Physical Review Letters 96 (7) (2006). paper number: 075502.

[38] A. Saai, H. Louche, L. Tabourot, H. Chang, Mechanics of Materials 42 (3) (2010) 275-292.

[39] M. Sauzay, Comptes Rendus Mécanique 334 (6) (2006) 353-361.

[40] P. Schlosser, H. Louche, D. Favier, L. Orgeas, Strain 43 (2007) 260-271.

[41] J.C. Simo, R.L. Taylor, Computer Methods in Applied Mechanics and Engineering 48 (1) (1985) 101-118.

[42] G.I. Taylor, H. Quinney, Proceedings of the Royal Society of London. Series A Containing Papers of a Mathematical and Physical Character 143 (849) (1934) 307-326.

[43] L. Vincent, European Journal of Mechanics - A/Solids 27 (2) (2008) 161-180. 